\title{
Einige Feststellungen zur Psychotherapie in der obligatorischen Krankenpflegeversicherung (OKP): gesundheitspolitische Aspekte
}

Während für uns alle psychotherapeutische Angebote in der Behandlung psychisch kranker Menschen ihren festen Platz haben, zielen Bemerkungen und einzelne plakative Aussagen gewisser politischer Exponenten darauf ab, gerade diese in ihrer Wirksamkeit unbestrittenen Leistungen in Frage zu stellen. Psychotherapie ist integraler Bestandteil jeder Behandlung psychischen Leidens, sie kostet nicht nur, sondern vermindert langfristig gesehen soziale Folgekosten wie lange Arbeitsunfähigkeiten, Invalidität und Überbeanspruchung anderer medizinischer Leistungen, aber auch die Belastungen von Familien und Gemeinden nehmen ab. Psychisch kranke Menschen brauchen angepasste und individuell wählbare psychiatrisch-psychotherapeutische Angebote, sie haben ein Anrecht auf eine adäquate Behandlung ihrer Krankheit. Die psychiatrischen Fachgesellschaften sehen es als eine ihrer Hauptaufgaben an, sich für diese Rechte ihrer Patienten einzusetzen.

Dr. med. Hans Kurt, Präsident SGPP und FMPP

\section{Rudolf Balmer}

* Mitglied der Arbeitsgruppe Psychotherapie der FMPP
Korrespondenz:

Dr. med. Rudolf Balmer Facharzt FMH Psychiatrie und Psychotherapie

Martinsgasse 6

CH-4051 Basel

Tel. 0616921032

rudolf.balmer@magnet.ch
Im Sommer 2005, anlässlich der Debatte um die Alternativmedizin, kündigte der damalige Vizepräsident des BAG, Dr. med. H. H. Brunner, die Überprüfung der Psychotherapie als Pflichtleistung in der OKP an. Dies war überraschend, weil kein dringender gesundheitspolitischer Handlungsbedarf vorlag. Die Psychotherapie ist durch eine Verordnung nach den Kriterien von Wissenschaftlichkeit, Zweckmässigkeit und Wirtschaftlichkeit (WZW) geregelt (KVL Abs. 2). Die heute geltende Verordnung vom 29. September 1995, welche durch eine breitabgestützte Expertenkommission unter Prof. Kind (Zürich) erarbeitet wurde, hat zu keinem Kostenschub in diesem Bereich des Gesundheitswesens geführt. Die bestehende Unterversorgung an Psychotherapie ist weniger Ausdruck dieser Regelung als anderer Strukturprobleme in der psychiatrisch-psychotherapeutischen Versorgung der Schweiz [1]. Anfang 2006 liegen vom BAG noch immer keine offiziellen Informationen zum Vorgehen vor, ausser dass das «Dossier Psychotherapie» bearbeitet werde.

Die Probleme, die mit dieser Überprüfung der Psychotherapie als Pflichtleistung der OKP auf- geworfen werden, sind nicht allein wissenschaftlicher Natur. Die Beurteilung der Psychotherapien nach den Kriterien von WZW enthält eine grundlegende gesundheitspolitische Dimension. Der gegenwärtige Prozess ist daher nicht nur eine fachwissenschaftliche, sondern auch eine Angelegenheit der Politik. In diesem Sinne ist es eine Notwendigkeit, dass sich politische Gremien damit befassen.

Auch wenn es zum gegenwärtigen Zeitpunkt noch keine offiziellen Informationen gibt und die Fachverbände (wie z.B. die FMPP [3]) noch nicht in den Beratungsprozess einbezogen wurden, so zeichnen sich doch gewisse Intentionen ab. So hat der Vizedirektor des BAG in Zeitungsinterviews bereits erklärt [3], dass es darum gehe, die Leistungen zu begrenzen und auf Kurztherapien zu beschränken. Eine ähnliche programmatische Erklärung ist im Kommentar des BAG in der Vernehmlassung zum PSY-Gesetz [5] enthalten; ein wichtiges Ziel dieses Gesetzes sei eine Senkung der Dauer und damit der Kosten im Bereich der Psychotherapie. In einem kürzlich veröffentlichten, im Auftrag des BAG erstellten Berichts des OBSAN [6] sind diese Gesichts- 
punkte zentral. Obwohl der Gegenstand dieses Berichtes, die Zulassung der psychologischen Psychotherapeuten/-innen zur OKP, noch nicht zuoberst auf der politischen Agenda liegt, so sind doch zwei im Bericht enthaltene Momente interessant. Dem Bericht ist ein Begriff von Wirksamkeit von Psychotherapiemethoden unterlegt, der sich an einem einseitigen Wissenschaftsverständnis orientiert, der hauptsächlich auf statistischer Datenerfassung basiert und der das breite, differenzierte Feld der Methodologien in der Psychotherapieforschung nicht berücksichtigt [7]. Er stellt als Konklusion fest: Kostenneutralität, eine Vorgabe des BAG, könne nur durch eine Systemänderung des KVG (u. a. durch Vertragsfreiheit) erreicht werden. Es liegt auf der Hand, dass damit gesundheitspolitische Fragen angesprochen sind.

\section{Ausbau der Psychiatrie und Psychotherapie}

Epidemiologische Untersuchungen zeigen eine hohe Häufigkeit von psychischen Störungen und Erkrankungen (40-50\% Lebenszeithäufigkeit); gleichzeitig wird auch ein quantitativer Mangel an psychiatrischen und psychotherapeutischen Angeboten festgestellt [1]. Dies gilt nicht nur für die psychischen Krankheiten und Störungen im engeren Sinne, sondern auch für vielfältige psychische Problemstellungen, die in der somatischen Medizin auftreten.

In einer im Jahr 2005 in der EU abgeschlossenen, auch für die Schweiz geltenden Studie [2] wird festgehalten: «Im Laufe eines jeden Jahres erleiden $27 \%$ der EU-Bevölkerung mindestens eine psychische Störung wie z.B. eine Depression, bipolare Störung, Schizophrenie, Alkoholoder Drogenabhängigkeit, Sozialphobie, Panikstörung, Generalisierte Angst, Zwangsstörungen, somatoforme Störungen oder Demenz. Das Lebenszeitrisiko, irgendwann einmal an einer psychischen Störung zu erkranken, liegt allerdings mit über 50\% der Bevölkerung wesentlich höher! Ausmass und Folgen sind dabei höchst variabel: Einige erkranken nur episodisch kurzzeitig über Wochen oder Monate, andere längerfristiger. Etwa 40\% der Betroffenen haben chronische Verläufe (über Jahre hinweg oder gar von der Adoleszenz bis an ihr Lebensende).

Mit geringen Unterschieden zwischen den EU-Ländern erhält nur etwa jeder vierte Betroffene mit psychischen Störungen irgendeine und noch weniger eine adäquate Behandlung. Oft vergehen viele Jahre und manchmal Jahrzehnte, bevor eine erste Behandlung eingeleitet wird. Ausnahmen sind Psychosen, schwere Depressionen und komplexe komorbide Muster.

\section{La psychothérapie dans I'assurance obligatoire des soins (AOS). Quelques aspects et constatations en matière de politique sanitaire}

Actuellement, I'OFSP vérifie les prestations de psychothérapie dans l'assurance obligatoire des soins. Selon les premières informations reçues, les prestations seront limitées et se concentreront sur les psychothérapies de courte durée. Dans ce contexte, le présent article soulève des questions fondamentales de politique sanitaire. La couverture en soins psychiatriques et psychothérapeutiques est insuffisante en Suisse, il faudrait plutôt étendre ces prestations. Par ailleurs et pour une prise en charge équitable, toutes les méthodes thérapeutiques devraient être prises en compte et les psychothérapies devraient proposer un mélange approprié de thérapie de brève et de longue durée. En effet, des études ont montré que les psychothérapies, même celles de longue durée, étaient avantageuses et efficaces à long terme. Avant que l'ordonnance en vigueur (OPAS $2^{\mathrm{e}}$ al.) ne soit modifiée, il faudrait procéder à une analyse approfondie du rapport coûts-efficacité, qui prenne en compte les économies réalisées au moyen des psychothérapies en ce qui concerne les coûts sociaux.

Unbehandelt verlaufen viele psychische Störungen häufig chronisch mit zunehmenden Komplikationen. Die besorgniserregend niedrige Behandlungsrate von psychischen Störungen, die in keinem anderen Bereich der Medizin in diesem Ausmass zu beobachten ist, kann nicht allein mit der immer noch den psychischen Störungen anhaftenden Stigmatisierung erklärt werden.»

\section{Feststellung}

Es ist aus gesundheitspolitischen Überlegungen ein dringendes Anliegen, die psychiatrischen und psychotherapeutischen Angebote und die Finanzierung im Rahmen der OKP nicht zu beschränken, sondern auszubauen. 
Die Psychotherapieangebote müssen differenziert sein - verschiedene Psychotherapieverfahren sind notwendig

Von etwa 1996 bis 1999 hat die vom BSV (Vorläufer des BAG) eingesetzte «Kommission Mattanza» in einem ausgewogenen Prozess, innerhalb dessen am 25. November 1997 auch eine breite Fachtagung stattgefunden hat, einen Bericht erarbeitet mit dem Titel «Kriterien zur Standardisierung der Prüfung von Wirksamkeit, Zweckmässigkeit und Wirtschaftlichkeit von Psychotherapiemethoden» [8]. Fazit dieses Berichtes ist nicht nur, dass sorgfältige Evaluationsprozesse stattfinden müssen, sondern auch, dass eine Vielfalt bezüglich der Psychotherapiemethoden sinnvoll ist und dass zur Überprüfung der Methoden differenzierte wissenschaftliche, den jeweiligen Methoden angepasste Kriterien eingesetzt werden müssen.

Die heute andeutungsweise veröffentlichten Ergebnisse der Evaluation durch das BAG weisen darauf hin, dass ein eher enger Wissenschaftsbegriff vorherrscht. Oberstes Kriterium scheinen Untersuchungen vom Typus der «kontrollierten Doppelblindstudien» (RCT = randomised controlled trial) zu sein, die aus der Medikamentenprüfung stammen. In ausführlichen Kritiken [9] haben verschiedene international renommierte Wissenschafter darauf hingewiesen, dass diese Form der Untersuchung von Psychotherapien gar nicht anwendbar ist. Vielmehr werden heute «naturalistische Studien» (Psychotherapiestudien im Feld der gewachsenen Versorgung) als massgebend angesehen. Eine Reihe solcher Studien verweist auf die Notwendigkeit eines differenzierten Angebotes [10].

Ein bedeutender Zweig der Psychotherapieforschung befasst sich damit, dass ein hoher Anteil an Wirksamkeit unabhängig von der Methode zustande kommt und dass ein wichtiger Anteil bei der gesundheitlichen Besserung durch die Qualität der Beziehung zwischen Patient/in und Psychotherapeut/in bedingt ist. Man spricht in der Forschung von der «Passung» der Partner in einem gemeinsamen Psychotherapieprozess, in dem ein aktives Mitmachen des Patienten Voraussetzung ist. Es liegt auf der Hand, dass in unserer pluralistischen Gesellschaft ganz unterschiedliche Zugänge in der Psychotherapie zu einer geglückten «Passung» beitragen.

Die Schweiz hat eine lange Psychotherapietradition, in der immer wieder verschiedene Psychotherapieverfahren entwickelt und überprüft worden sind. Die Entwicklung geht von Pionieren wie M. Bleuler, C. G. Jung, L. Binswanger und G. Benedetti aus (um nur einige wenige Exponenten zu nennen), und aus dieser Entwicklung ist die Psychoanalyse nicht wegzudenken. In den letzten Jahrzehnten gibt es eine Vielzahl von Forschern und Praktikern, die sich ernsthaft mit der Wirksamkeit der Psychotherapien auseinandersetzen. Im Zuge dieser Entwicklung sind auch Institute und Weiterbildungsgänge entstanden, die aus einem breiten Erfahrungswissen schöpfen und bemüht sind, die Patientenbedürfnisse abzudecken. Diese gewachsene Struktur gilt es in der weiteren Ausgestaltung der psychotherapeutischen Versorgung zu berücksichtigen.

\section{Feststellung}

Es ist aus wissenschaftlichen Gründen notwendig, dass im Psychotherapiesektor eine Methodenvielfalt gepflegt wird. Es ist erwiesen, dass Psychotherapieergebnisse wesentlich besser sind, wenn die Wahl der Behandlungsmethode der individuellen, gesunden Verarbeitungsweise eines Patienten entspricht und er einen eigenen Bezug zur Methode finden kann. Dies setzt eine Wahlmöglichkeit voraus.

\section{Eine ausgewogene Psychotherapie- versorgung muss Kurz- und Langzeit- verfahren anbieten können}

Es wird in Fachkreisen davon ausgegangen, dass rund zwei Drittel aller Patienten mit psychischen Störungen mit Kurztherapien behandelt werden können. Es handelt sich dabei um Patienten mit eher umschriebenen Störungen (z.B. gewisse Angststörungen), oder es handelt sich um Patienten in Lebenssituationen, die eine längere Behandlung nicht erlauben. Umstritten ist in Fachkreisen, was unter «Kurztherapie» zu verstehen ist. Als Kurztherapien im engeren Sinne können Therapien von 10 bis 20 Sitzungen, als kürzere Therapien solche von 40 bis 100 Sitzungen bezeichnet werden. Eine Langzeittherapie ist eine Behandlung, die über 100 Sitzungen hinausgeht und häufig auch in einer höheren Stundenzahl pro Woche durchgeführt wird.

In Umkehrung der obigen Aussage muss davon ausgegangen werden, dass mindestens ein Drittel der Menschen mit psychischen Störungen längere Therapien von über 100 Sitzungen benötigt.

Es gibt einzelne Aussagen [4], dass Psychotherapien über 60 Sitzungen hinaus wirkungslos seien. Solche Aussagen können wissenschaftlich nicht gehalten werden, zumindest nicht für psychoanalytische Behandlungen. Verschiedene Forscher (zwei umfassende Untersuchungen: $[10,11])$ haben sich eingehend mit Langzeitbehandlungen befasst. Die Studien belegen deut- 
liche Verbesserungen (weniger Beschwerden, bessere Lebensbewältigung) mit zunehmender Dauer der Behandlungen. Sie zeigen auch eine nachhaltige Wirkung, indem diese Verbesserungen nach Abschluss der psychoanalytischen Behandlungen noch zunehmen.

Der heutige Trend zu Kurztherapien und die Aussagen, dass weitgehend nur diese hilfreich seien, wurde aus ökonomischen Gründen vor Jahren in den USA lanciert. Eine Korrektur brachte 1995 der «Consumer Report» von Seligman. In der Konsumentenbefragung wurden nicht in erster Linie die Kurztherapien als hilfreich empfunden, sondern es wurde deutlich, dass bei längeren Behandlungen, die ohne Eingriff von aussen beendet werden konnten, bedeutend grössere Behandlungschancen gegeben sind [12]. Im Jahre 2003 wurde die gleiche Studie in Deutschland wiederholt [13] mit dem Ergebnis, dass zwischen psychoanalytischen und verhaltenstherapeutischen Verfahren nur marginale Unterschiede feststellbar waren. Zudem: «...je länger die Behandlungen erfolgt waren, desto grösser fielen die Besserungsraten aus.»

\section{Feststellung}

Eine angemessene Versorgung muss ein ausgewogenes Angebot von Kurz- und Langzeitpsychotherapien aufweisen. Es ist zu beachten, dass sich die heute geltende Regelung gerade in dieser Hinsicht bewährt und zu differenzierten Dauern von Psychotherapien geführt hat. Es wäre politisch kurzsichtig, wenn durch die Forcierung von Kurztherapien Rahmenbedingungen geschaffen würden, welche die Motivation der Menschen, sich mit ihren Schwierigkeiten ernsthaft zu befassen, untergrüben.

\section{Psychotherapie ist kostensparend}

Gegenwärtig werden für die ambulanten psychotherapeutischen Leistungen rund 550 Millionen Franken ausgegeben. Diese Ausgaben entsprechen rund 1,5\% der gesamten Ausgaben oder 5\% der ambulanten Ausgaben (Zahlen von 2000; die Zahlen dürften für das Jahr 2005 proportional nicht völlig verändert sein).

\section{Feststellung}

Diese Ausgaben, gemessen an der Häufigkeit psychischer Störungen und Krankheiten, sind eher zu gering und dokumentieren eine Unterversorgung.

In verschiedenen Verlautbarungen hat das BAG ausgeführt, dass ein wichtiges Ziel sei, die Dauer der Psychotherapien zu kürzen, um damit die Kosten zu senken.

\section{Feststellung}

Eine solche Zielformulierung greift gesundheitspolitisch offensichtlich zu kurz. Abgesehen davon, dass es aus ethischen Gründen eine Selbstverständlichkeit ist, dass bei psychischen Störungen von Krankheitswert auch in chronischen Fällen psychiatrische und psychotherapeutische Behandlungen vorgenommen werden müssen, so weisen doch verschiedene wissenschaftlich abgesicherte Befunde darauf hin, dass auch längerdauernde Psychotherapien insgesamt kostensparend sind. Ein Druck auf die Verkürzung der Therapien verstärkt die Tatsache, dass Patienten viel zu oft unbeendete Therapien abbrechen und zu resignierten «Drehtürpatienten» werden. Die «Konstanzer Studie» über die Kosten ambulanter psychotherapeutischer Behandlungen weist nach, dass diese innerhalb weniger Monate ihre Kosten durch Einsparungen anderer medizinischer Leistungen wieder einspielen [7].

Wer in der Praxis mit Menschen mit psychischen Störungen zu tun hat, weiss, welche starken Auswirkungen diese Störungen auf das Leben der Betroffenen haben: Es ist die gesamte Gesundheit, die Arbeitsfähigkeit und auch das Leben der umgebenden Menschen in Arbeit und Familie über Generationen hinweg betroffen. Die Erfahrung belegt, dass Psychotherapie in diesen Aspekten hilfreich eingreift und letztlich Folgekosten vermeidet. Bei einer willkürlichen Kürzung der Behandlungsdauer ist $\mathrm{zu}$ erwarten, dass die Versicherten immer wieder Therapietranchen (in psychotherapeutischen und in anderen medizinischen Bereichen) beanspruchen müssen, was letztlich nicht billiger zu stehen kommt, als wenn bei gegebenen Indikationen längere Psychotherapien durchgeführt werden. Die Basis für weitreichende gesundheitspolitische Entscheide wären umfassende Kosten-Nutzen-Rechnungen, die auch die Folgekosten in anderen Bereichen berücksichtigen (z. B. in der übrigen Medizin, im Sozialbereich, bezüglich Arbeitsfähigkeit, Invalidität, bezüglich sozialer Kosten für die Kinder psychisch kranker Menschen usw.).

\section{Feststellung}

Die «Konstanzer Studie» [7] zeigt auf, wie vertiefte psychotherapeutische Arbeit körperliche Erkrankungen, Chronifizierungen und Invalidisierungen und damit Zusatzkosten vermeiden hilft. Solche umfassende Kosten-Nutzen-Studien gibt es derzeit in der Schweiz nicht. Es wäre gesundheitspolitisch von grösster Priorität, dass solche Studien in Auftrag gegeben würden. 
Auch die gesundheitlichen und finanziellen Auswirkungen eines auf Kurztherapien beschränkten Psychotherapieangebotes sind ungenügend erforscht. Entscheide bezüglich des Leistungskataloges erfordern aber solche Grundlagen.

Die Datenlage bezüglich der Wirtschaftlichkeit von Psychotherapien ist unvollständig, aber verglichen zur übrigen Medizin nicht schlechter. Bei Entscheiden über die Psychotherapie dürfen nicht striktere Kriterien als in der somatischen Medizin angewandt werden. Sie können erst gefällt werden, wenn die Datenlage besser ist.

\section{Systemänderung des KVG für die Psychotherapie?}

Im Bereich der psychotherapeutischen Versorgung wird zunehmend über systemverändernde Massnahmen nachgedacht, z.B. Vertragsfreiheit zwischen kostengünstigsten Anbietern und Krankenversicherern, Einbezug in Versorgungsnetzwerke oder Verlagerung der Psychotherapie in den Bereich der Zusatzversicherungen. Es sei hervorgehoben, dass bezüglich des gesundheitlichen und volkswirtschaftlichen Nutzens von Psychotherapien zwischen dem Gemeinwesen, den Patienten und den Versicherern erhebliche Interessensunterschiede und -konflikte bestehen. Für eine allfällige «Vertragsfreiheit» müssten daher klare gesundheitspolitische Rahmenbedingungen festgelegt werden, welche die Patientenbedürfnisse schützen. Es ist in diesem $\mathrm{Zu}$ sammenhang besonders $\mathrm{zu}$ betonen, dass für den psychisch kranken Menschen der «freie Markt» noch viel weniger spielen kann als für Patienten in der somatischen Medizin. Psychotherapie ist ein zentraler Bestandteil jeder Behandlung von psychischen Erkrankungen und psychosomatischen Problemstellungen. Es ist über Fachkreise hinaus unbestritten, dass Psychotherapie ein integrierter Bestandteil der psychiatrisch-psychotherapeutischen Angebote ist und damit auch Teil der OKP zu sein hat.

\section{Literatur}

1 Eine ausführliche Analyse liegt im Bericht Nationale Gesundheitspolitik Schweiz - Psychische Gesundheit, OBSAN 2002, vor.

2 Wittchen H-U. Psychische Störungen in Deutschland und in der EU. Grössenordnung und Belastungen. Pressemitteilung, 1.12.2005. www.psychologie.tu-dresden.de.

3 FMPP ist die Dachorganisation der SGPP (Schweizerische Gesellschaft für Psychiatrie und Psychotherapie) und der SGKJPP (Schweizerische Gesellschaft für Kinder- und Jugendpsychiatrie und -psychotherapie).
4 NZZ am Sonntag, 11. Dezember 2005.

5 Vernehmlassungsentwurf zum Eidgenössischen Gesetz der Psychologieberufe (Abkürzung: PSY-Gesetz).

6 Spycher S, Margraf J, Meyer PC. Zulassung der psychologischen Psychotherapeut/innen zur Krankenversicherung? Arbeitsdokument Nr. 15. Neuchâtel: OBSAN; November 2005.

7 Der Bericht zitiert die «Konstanzer Studie» nicht, die im Auftrage der DGPT (Deutsche Gesellschaft für Psychoanalyse, Psychotherapie, Psychosomatik und Tiefenpsychologie e.V.) erstellt wurde und die nachweist, dass die ambulanten psychoanalytischen Psychotherapien innerhalb weniger Monate ihre Kosten durch Einsparungen anderer medizinischer Leistungen wieder einspielen (Substitutionseffekt der Kosteneinsparung von Psychotherapien). Breyer F, Heinzel R, Klein T. Kosten und Nutzen ambulanter Psychoanalyse in Deutschland. Gesundheitsökonomie und Qualitätsmanagement 1997;2:59-73.

8 BSV, Bundesamt für Sozialversicherung. Kriterien zur Standardisierung der Prüfung von Wirksamkeit, Zweckmässigkeit und Wirtschaftlichkeit von Psychotherapiemethoden, formuliert von Dr. med. G. Mattanza. 5. Entwurf. Februar 1998.

9 England: Fonagy P. Process and outcome in mental health care delivery: A model approach to treatment evaluation. Bull Menninger Clin 1999;63: 288-304; Deutschland: Leichsenring F, Rüger U. Psychotherapeutische Verfahren auf dem Prüfstand von Evidence Based Medicine (EBM). Z Psychosom Med Psychother 2004;50:203-17. In diesem Artikel gibt es eine differenzierte Argumentation über angemessene Psychotherapieforschung.

10 Leuzinger-Bohleber M, Stuhr U, Rüger B, Beutel M. Langzeitwirkungen von Psychoanalysen und Psychotherapien - eine multiperspektivische, repräsentative Katamnesestudie. Psyche 2001;55: 193-276.

11 Sandell R, Blomberg J, Lazar A. When reality doesn't fit the blueprint: doing research on a psychoanalysis and psychotherapy in a public health service programme. Psychother Res 1997;7:333-44. Siehe auch in diesem Zusammenhang: Deutsche Psychoanalytische Vereinigung. Indikation und Wirksamkeit, Psychoanalyse und psychoanalytische Verfahren in der medizinischen Versorgung. 2001 (Ausgabe für die Schweiz: SGPsa. 2004) sowie EFPP Schweiz. Die Psychoanalytische Psychotherapie. Argumentarium, Grundlagen, therapeutische Wirkungen, Wirtschaftlichkeit, aktueller Forschungsstand. Basel: Selbstverlag; 2000.

12 Seligman MEP. The effectiveness of psychotherapy. Am Psychol 1995;50:965-74.

13 Hartmann S, Zepf S. Effectiveness of psychotherapy in Germany: a replication of the consumer-reports-study. Psychother Res 2003;13: 235-42. 